Periostes nicht die ganze Dicke der knöchernen Schädeldecke durchmeisselt wird, wie dies Wagn er thut, sondern dass mittels eines scharfen Meissels nur die Tabula externa im Umfange des Hautperiostlappens abgemeisselt und an seiner Basis eingebrochen wird. Darauf wird der so gebildete Hautperiostknochenlappen, der also nur die 'labula externa enthält, umgeklappt, und der Rest des Schädeldaches entfernt. Von Köni ${ }^{1}$ ) wurde dieses Verfaluren weiter ausgebildet, so dass es sich zum Ersatze grösserer Schädeldefecte eignete. König hatte einen $8 \mathrm{~cm}$ langen und $5 \mathrm{~cm}$ breiten Knochendefect der linken Schläfen- und Sclieitelbeingegend bei einem 30jährigen Manne zu decken, der nach einer complicirten Schädelfractur entstanden und für den Patienten die Quelle zahlreicher physischer wie psychischer Störungen geworden war, Zum Zwecke der Deckung umschnitt er zunächst die Weichtheile am Rande des knöchernen Defectes bis auf eine $1 \frac{1}{2} \mathrm{~cm}$ breite Stelle, die später zur Ernährung des Weichtheillappens dienen sollte. Darauf nahm er die Maasse des Defectes und umschnitt oberhalb des Defectes einen Ersatzlappen mit breitem, dem ersten gegenüberliegenden Stiel, der in seiner Peripherie überall einen Centimeter breiter war als die Lücke im Schädel und durch einen Sporn intacter Haut von dieser getrennt blieb. Nach erfolgter Retraction der Haut und des Periostes schälte er im ganzen Umfange des Weichtheillappens mittels eines scharfen Meissels eine knöcherne Schale $a b$ und präparirte dann die umsehnittene Narbe von dem Gehirn bis auf den erwähnten $1 \frac{1}{2} \mathrm{~cm}$ breiten Stiel ab. Jetzt wurden die beiden Lappen mit einander vertauscht, derart, dass der Lappen, der die Narbe enthielt, an die Stelle des neugebildeten Hautperiostknochenlappens und dieser an die Stelle des Schädeldefectes zu liegen kam. Der Hautperiostknochenlappen wurde an dem Orte seiner neuen Bestimmung durch Periost- und Hautnähte flxirt und bewirkte hier, obschon der Knochentheil desselben bein Abmeisseln mehrfach eingebrochen war und nur eine relativ dünne Knochenschicht enthielt, innerhalb von sechs Wochen einen vollkommen festen und sicheren Verschluss des früher vorliandenen Defectes, an den man bei der Entlassung nur durch ein schwaches Pulsiren am unteren Ende des Ersatzlappens erinnert wurde. Die früher vorhandenen krankhaften Geliirnerscheinungen waren völlig beseitigt.

Die geschilderte Methode ist zum Ersatze grösserer Schädeldefecte bisher mehrmals von anderen mit dem gleichen, guten Erfolge versucht worden. So stellte Schönborn ${ }^{2}$ ) dem 20 . Chirurgencongress einen 18jährigen Mann vor, bei dem er einen $14 \mathrm{~cm}$ langen und 2-4 cm grossen, unregelmässigen Defect, der beide Stirnbeine betraf und nach complicirter Fractur entstanden war, auf die angegebene Weise gedeckt hatte. Scliönborn hatte die Narbe völlig exstirpirt und deckte den durch Wegnahme des Hautperiostknochenlappens entstandenen Defect mittels T'ransplantationen nach Thiersch. Da der Ersatzlappen, der auf die Stirn zu liegen kam, aber dem behaarten Kopfe entnommen war, so tauschte er in einer späteren Operation aus kosmetischen Gründen die Lappen, ohne die daruntergelegenen Knochen mitzunehmen, aufs neue, so dass der durch die Transplantation gedeckte Weichtheildefect an die Stelle des frïheren Schädeldefectes und der behaarte Theil des Hautperiostknochenlappens wieder an seine alte Stelle z1 liegen kam.

Weitere Mittheilungen über gelungene osteoplastische Deckungen von Schädeldefecteu liegen vor von $\mathrm{H}$. $\mathrm{Braun}^{3}$ ), von Tietze $\mathrm{e}^{4}$ ) aus der Mikulicz'schen Klinik, ferner von Riegne ${ }^{5}$ ) und B. Seajme $r^{6}$ ). Czerny berichtete auf dem 21. Chirurgencongress über einen Fall von Hirntumor, bei dem er die Mülle r'sche Methode anwandte.

In Anbetracht dieser immerhin noch spärlichen Casuistik hat es vielleicht Interesse und Werth, den aufgezähIten Fällen noch einen weiteren Fall anzureilıen, der auf der äusseren Abtheilung des städtischen Krankenhauses am Urban zur Beobachtung kam.

Siebenjähriger Knabe Heinrich Sch., aufgenommen 4. Juli 1891

V. Aus dem Städtischen Krankenhause am Urban in Berlin, Abtheilung des Herrn Director Dr. Körte.

\title{
Ueber traumatische Schädeldefecte und ihre Deckung.
}

Von Dr. Adolf Brentano, Assistenzarzt.

(Fortsetzung aus No. 17.)

Weit häufiger wird man zur Deckung traumatischer Schädeldefecte auf die M:üller-König'sche Methode angewiesen sein. Müller ${ }^{1}$ ) hat im Jahre 1890 ein Verfahren zur Eröffnung des intacten Schädels angegeben, das eine Modification des oben geschilderten Wagner'schen darstellt und darin besteht, dass nach Umschneidung eines bogenförmigen Weichtheillappens und des

1) W. Máller, Zur Frage der temporären Schädelresection an Stellè der 'l'repanation. Centralblatt für Chirurgie 1890, No. 4.

1) F. König, Der knöcherne Ersatz grosser Schädeldefecte. Centralblatt für Chirurgie 1890, No. 27.

2) Schonborn, Ein Fall von knöchernem Ersatze eines grossen Schädeldefectes nach der Methode von König. Verhandlungen des Congresses der deutschen Gesellschaft für Chirurgie 1891.

3) H. Braun, Exstirpation eines den Schädel perforirenden Hautcarcinoms bei einem 14jährigen Mädchen. Verhandlungen des Congresses der deutschen Gesellschaft für Chirurgie 1892.

$\left.{ }^{4}\right)$ ' Tietze, Vorstellung zweier. Patienten mit osteoplastischem Verschlusse von Schädeldefecten. ibidem.

5) 0. Riegner, Trepanation wegen subduralen Blutergusses mit späterem osteoplastischem Ersatze des Schädeldefectes nach Konig. Deutsche med. Wochenschrift 1893, No. 28 .

6) B. Seajmer, Ein Fall von Meningocele spuria traumatica mit osteoplastischem Verschlusse des Defectes. Wiener klin. Wochenschrift 1893, No. 12 . 
die von einem Sturz von einer Leiter herrührte. Die Splitter stark untereinandergesehoben theilweise ganz ausser Zusammenhang mit dem Perioste. Von : der Bruchstelle gehen Fissuren namentlich nach hinten. Vollige Bewusstlosigkeit, 136 regelmässige Pulse. Temperatur im Rectum 37,1. In Chloroformnarkose (Operateur: Dr. Si e g mund) Erweiterung der strahlenförmigen Wunde. Entfernung eines dreieckigen, ganz isolirten Knochensttuckes von $4 \frac{1}{2} \mathrm{~cm}$ Länge und $3 \mathrm{~cm}$ Breite und mehrerer kleinerer Stticke nach Lösung derselben durch Randabmeisselung. Dura àn einer erbsengrossen Stelle verletzt. Desinfection. Tamponade der ganzen Wunde mit Jodoformgaze. Durawunde mit einem Jodoformgazestreifen drainirt. Alsbald nach der Operation Wiederkehr des Bewusstseins. Keine nachweisbaren Störungen der Sensibilität oder Motilität.

Am nächsten Tage höchste Temperatur 38,9, Puls 172 (Jodoformwirkung?). Allmähliches Sinken der Pulsfrequenz und der Temperatur Bei reactionslosem Wundverlaufe am 9. Juli Secundärnaht des Periostes und der Haut. Die Nähte halten nur zam Theil und mlissen theilweise am 14. Juli entfernt werden.

Am 21. Juli, als die Wunde gut granulirte, neue Naht, die eine erhebliche Verkleinerung bewirkt. Der Knochendefect bleibt deutlich fühlbar, hat eine leicht S-förmige Gestalt, misst in seiner grossten Länge 8, in seine grossten Breite $3 \mathrm{~cm}$ und nimmt das Stimbein und einen kleinen Theil des rechten Scheitelbeines ein. Deutliche Gehirnpulsationen an der Stelle des Defectes.

Am 16. September in Chloroformnarkose (Operateur: Direktor Dr. Körte) osteoplastischer Verschluss des Defectes nach König. Umschneidung der Weichtheilnarbe, die den Knochendefect ausfüllt. Abpräparirung derselben von der Dura, ohne dass diese dabei eröffnet wird. Ein nach dem Gesichte zu liegender Stiel wird erhalten. Oberhalb und nach innen zu von dem so gebildeten Lappen wird der Ersatzlappen mit nach hinten zu gelegener Basis umschnitten, so dass er an Grösse allseitig den Schädeldefect ibertrifft und von letzterem durch einen Sporn unversehrter Haut getrennt bleibt. Der umschnittene Ersatzlappen wird nach Retraction der Haut und des Periostes mittels eines scharfen Meissels innerhalb der Tabula externa abgetrennt, dann beide Lappen vertauscht und an dem Orte, wo sie einheilen sollen, durch Seidennähte fixirt. Da der Lappen, welcher die Narbe enthielt, nicht vollständig den Defect ausfullte, der durch Wegnahme des Hautperiostknochenlappens entstanden war, so wird ein dritter Lappen medianwärts von letzterem mit nach oben und hinten gerichtetem Stiel gebildet und zur Bedeckung des noch blossliegenden Knochens verwendet. Die durch Bildung des dritten Lappens gebildete Wunde wird mit Thiersch'schen Transplantationen bedeckt. Reactionsloser Verlauf.

Am 29. September 1891 werden sämmtliche Nähte entfernt. Prima intentio erfolgt. Früherer Schädeldefect fest verschlossen. Nur die ausserste Spitze des ubergepflanzten Hautperiostknochenlappens ist nekrotisch geworden und infolgedessen an einer circumscripten, etwa funfpfennigstückgrossen Stelle noch Pulsation wahrzunehmen.

Am 15. October wird Patient entlassen, da die Erlaubniss zu einer Operation behufs Deckung des kleinen Defectes von den Eltern verweigert wurde.

Am 26. Februar 1893 stellt sich Patient wieder vor, und es konnte festgestellt werden, dass der frühere Defect fast mit Knochensubstanz ausgefüllt war. Nur einen Finger breit ubber dem Margo supraorbitalis fithlt man noch an einer eben die Fingerkuppe aufnehmenden Stelle Pulsation, ausserdem findet sich noch am oberen Rande des fruheren Defectes eine ca. $2 \mathrm{~mm}$ breite Fissur, wo man bei genauerem Hinsehen Pulsation sieht, aber nicht ftihlen kann.

Eine besondere Methode der Autoplastik hat Seyde ${ }^{1}$ ) angewandt, um einen grossen Knochendefect des Schädels zum Verschlusse zu bringen, zu einer Zeit als das Verfahren nach König noch nicht bekannt war. Um einen $5,5 \mathrm{~cm}$ langen, $4 \mathrm{~cm}$ breiten Defect des linken Scheitelbeines, der nach einer complicirten Depressionsfractur zurückgeblieben war, zu decken, meisselte Seydel zwölf Tage nach dem Trauma, als die Gehirnerscheinungen, die bis dahin bestanden hatten, verschwunden waren und der infolge des Traumas entstandene Querriss verklebt war, ein $5,5 \mathrm{~cm}$ langes und $4 \mathrm{~cm}$ breites Stück sammt dem zugehörigen Perioste aus der Corticalis der Tibia aus, legte es in schwache Kochsalzlösung, zertheilte es dann in 6-8 gleichmässige Stücke und füllte damit mosaikartig den Schädeldefect aus, ohne zunächst die Weichtheile darüber zu vernähen. Erst zehn Tage später, als er sich überzeugt hatte, dass die Stücke lebensfähig geblieben und fest mit der Dura verwachsen waren, deckte er dieselben durch einen dem Hinterhaupte entnommenen gestielten Lappen. Die implantirten Stücke hatte er in der Zwischenzeit mit Silk bedeckt und die Wunde darüber mit Jodoformgaze und trockenem aseptischem Verbande bedeckt gehalten.

Will man von keinem der bisher geschilderten Verfahren Gebrauch machen, so bleibt als letzte Möglichkeit, ausgedehntere traumatische Schädeldefecte zum Verschlusse zu bringen, die Implantation der gelösten und herausgenommenen Splitter übrig. Der erste, der auf diesem Wege traumatische Defecte ausfüllte, war Mac Ewen. Fussend auf den guten Resultaten, welche das Wiedereinheilen heraustrepanirter Stücke bei Thieren ergeben hatte und die er in mehreren Fällen auch beim Menschen bestätigen konnte, schlug

1) SeydeI, Eine neue Methode grosse Knochendefecte des Schädels zu decken. Centralblatt. f. Chir. 1889, p. 12. er vor, bei complicirten Brüchen des Schädels die Splitter zur Ausfüllung des Defectes in ähnlicher Weise wieder zu benutzen. Im Jahre 18881) berichtete er, dass er die grossen Knochendefecte nach Schädelverletzungen sowohl, wie nach ausgedehnteren Trepanationen in der Regel durch Implantation zahlreicher kleiner Knochenstückchen zwischen Haut und Dura ausgefüllt und bei ungestörter Asepsis damit schliesslich stets vollständige Verknöcherung im Bereiche der Lücke erreicht habe.

So gross nun die Zahl der Fälle ist, bei denen die aus dem vorher intacten Schädel, sei es mit Hammer und Meissel; sei es mit der Trepankrone ausgelösten Knochenstücke in den Defect wieder eingesetzt wurden, so selten sind bei complicirten Schädelbrïchen die zertrümmerten Knochentheile zur Wiederausfüllung des Defectes benutzt worden. Es liegen nur Mittheilungen vor von Gerstein ${ }^{2}$ ), welcher ein $4 \mathrm{~cm}$ langes und $1,5 \mathrm{~cm}$ breites Knochenstück, das er entfernen musste, vorübergehend in schwache Sublimatlösung legte, um es schliesslich wieder einzupflanzen. Fr war dabei genöthigt, Theile desselben abzutragen, um es für die Lücke passend zu machen. Es heilte ein, und bei dem wenige Monate später an Pneumonie erfolgten Tode des Patienten konnte festgestellt werden, dass das Stück überall da, wo es mit Knochen in unmittelbarer Berührung gestanden hatte, knöchern verbunden war.

Von v. Bramann, $\left.{ }^{3}\right)$ der der chirurgischen Section der 64 . Versammlung deutscher Naturforscher und Aerzte drei Kranke mit complicirten Schädelbrïchen vorstellte, bei denen er vollständig gelöste Knochenstücke zur Einheilung brachte.

Von Jones. ${ }^{4}$ ) Er hatte die Knochenstücke in Carbolsäurelösung 1:30 aufbewahrt. In zwei Fäll en reactionslose Einheilung, in einem Falle Ausstossung der Knochenstücke unter Eiterung.

Die Wunde war in allen Fällen nach der Implantation völlig geschlossen worden.

Von Möller, ${ }^{5}$ ) der im ärztlichen Verein zu Hamburg einen achtjährigen Knaben vorstellte, bei dem ein ca. thalergrosses, durch Meisseltrepanation gelöstes Stück in den Defect wieder eingelegt wurde, während ein zweites etwa eben so grosses wegblieb. Das eingelegte Stück heilte reactionslos ein, obschon die Wunde infolge der Eröffnung der Stirnhöhle mit der Nasenhöhle in Verbindung stand.

Just $0^{6}$ ) erzielte die Einheilung eines $6-7 \mathrm{~cm}$ langen und $4-4^{1 / 2} \mathrm{~cm}$ breiten Knochenstückes des rechten Scheitelbeines bei eomplicirter Fractur.

Ferner berichtete in der sich an den Vortrag des Verfassers (in d. fr. chir. Vereinig. am 13. März 1893) anschliessenden Discussion Thieme (Cottbus) über mehrere Fälle glïcklich verlaufener frischer Implantationen.

Da die im städtischen Krankenhause am Urban bisher zur Behandlung gekommenen Fälle von traumatischen Schädeldefecten - mit Ausnahme des schon geschilderten Falles - sämmtlich durch Implantation der gelösten und herausgenommenen Trümmer mit bestem Erfolge zum Verschlusse gebracht wurden, so sind wir in der Lage, uns gerade iber diese Methode, bezüglich ihrer Brauchbarkeit vom klinischen : Standpunkte aus, ausführlicher äussern zu können. Es handelte sich in allen Fällen, die im folgenden geschildert werden; um complicirte Brüche des Schädeldaches mit Splitterung und Depression der Fragmente. Die Indication zum operativen Eingreifen war nicht in allen Fällen dieselbe, dagegen bot das Vorgehen bei der Ausfüllung des Defectes durch Implantation so wenig verschiedenes, dass wir eine Beschreibung derselben vorausschicken wollen, um spätere Wiederholungen zu vermeiden. Nach sorgfältiger Desinfection der Haut des rasirten Schädels wurde die Bruchstelle durch grosse Erweiterungsschnitte freigelegt, das Periost soweit als nöthig zurückgeschoben, und dann mit Hülfe von Hammer und Meissel die eingedrückten Stücke gelöst und herausgenommen (also ohne Periostbekleidung!), die einzelnen Stücke wurden dann in $1 \%$ Sublimatlösung durch Abreiben mit den Händen gereinigt und in wàrmer $0,75 \%$ Kochsalzlösung bis zu ihrer Wiedereinlegung aufbewahrt. Zeigten einzelne der Knochenstïcke, was selten der Fall war,

1) William Macewen, Ueber Chirurgie des Hirn und Rúckenmarks. Adresse gelesen auf der 56. Jahresversammlung der British. med. Association zu Glasgow am 9. August 1888. Referat im Centralblatt für Chirurgie 1888, No. 43.

2) Gerstein. Ueber. Verschluss von Defecten am Schädel. Verhandlungen des Chirurgencongresses 1889

3) V. Bramann, Centralblatt f. Chirurgie 1891, No. 48, p. 944

4) Jones, Three cases of compound depressed fracture of the skull in which the bones were replaced after beeing temporaly removed. Med. Chronicle 1890, December.

5) Moller, Deutsche med. Wochenschrift 1893, No. 48, p. 1285.

6) Justo, Sobre alginos casos de lesiones traumaticas de la bóveda del craneo y del cerebro. Rev. de la soc. méd. Argentina, Buenos-Aires 1893, März und April. Ref. im Centralbl. f: Chir. 1893, No. 41 
gröbere Verunreinigungen, die sich auf die geschilderte Weise nicht entfernen liessen; so wurde die betreffende Stelle mit der Lu.er'schen Zange abgekniffen.

Durawunden wurden zum. Zwecke besserer Desinfection erweitert und danach entweder vollkommen mit Catgut vernäht, oder an einer kleinen Stelle offen gelassen, von der aus Jodoformgaze unter die Nahtlinie und die Nachbarschaft der Wunde geschoben wurde.

Lagen Gehirnverletzungen vor, so wurden dieselben zunächst mittels kleiner Gazestückchen, die mit $1 \%$ Sublimat getränkt waren, ausgerieben, dann mit Jodoformgaze ausgetrocknet und stets drainirt. Zur Drainage dienten uns anfangs schmale Jodoformgaze-, später Silk protectif-Streifen, die bis auf den Grund der Wunde eingeführt wurden, ohne jemals dabei eine nennenswerthe Reaetion hervorzurufen. Selbstverständlich blieb die Dura über der Gehirnwunde stets offen, und auch bei der Implantation der Knochenstücke wurde die verletzte Gehirnpartie wenigstens soweit frei gelassen, dass die Wirkung der Drainage nicht beeinträchtigt wurde.

Ganz besondere Sorgfalt wurde stets auf die Stillung der Blutung verwandt. Gefässse der Dura wurden umstochen und unterbunden, Blutungen aus der Diploë durch vorübergehende Tamponade mit Jodoformgaze meist rasch zum Stehen gebracht, event. durch Anwendung von Glühhitze (Paquelin) gestillt.

Waren Dura- und Gehirnwunden auf diese Weise versorgt, so wurden die Knochenstücke aus der Kochsalzlösung heraus an den Ort ihrer Bestimmung gebracht, indem sie lose neben einander auf die Dura aufgelegt wurden. Sie wurden dabei nicht weiter verkleinert, sondern behielten die Grösse, die sie nach der Herausnahme aus der Bruchstelle hatten, bei, d. h. ungefähr die Grösse eines Daumennagels, manche etwas grösser, andere kleiner, wie das Trauma sie geformt hatte. Nach beendigter Implantation wurden die Weichtheile (Haut und Periost zusammen) über derselben durch weitläufige Seidennaht vereinigt bis auf einen oder zwei Wundwinkel, die event. zur Drainage dienten.

Es verdient hervorgehoben zu werden, dass es nach unseren Erfahrungen vollkommen gleichgültig für den definitiven Erfolg ist, ob die Knochenstücke jedesmal der ganzen Dicke der Schädeldecke oder nur der Tabula externa oder interna allein angehören, ferner erscheint auch die Grösse der eingelegten Stücke ohne Belang, da wir solche von $1-2 \mathrm{~cm}$ Breite und andererseits selbst Meisselspähne benutzten und einheilen sahen. Auch die Art der Einlegung der einzelnen Stücke beeinträchtigt nicht das gute Endresultat, es ist daher nicht nöthig, dieselben so zu lagern, dass die Dura jedesmal mit der glatten Innenseite der Tabula vitrea in Berührung kommt. Wir haben wiederholt auch rauhe, kantige Theile der Diploë direkt der Dura aufgelegt.

Von Wichtigkeit für das Gelingen der Implantation scheint dagegen eine streng durchgeführte Asepsis, eine möglichst exacte Blutstillung und eine sorgfältige Naht der 'Weichtheile über den eingepflanzten Knochenstücken zu sein. (Fortsetzung folgt.) 